\title{
Harnessing the power of healthy buildings research to advance health for all
}

\author{
Joseph G. Allen ${ }^{1} \cdot$ Michael S. Waring ${ }^{2}$
}

Received: 13 September 2019 / Accepted: 19 September 2019 / Published online: 2 December 2019

(c) The Author(s), under exclusive licence to Springer Nature America, Inc. 2019

The two forces of rapid population growth and urbanization are colliding and creating one of the greatest public health challenges, and opportunities, ever-our buildings. As consumers of $40 \%$ of energy globally, buildings contribute greatly to emissions of air pollutants and greenhouse gases, but also have the potential to mitigate emissions by way of efficient improvements. Buildings also influence our health directly from the time we spend in them, due to our exposure to air pollution of both outdoor and indoor origin, as well as their microbiomes.

The merging of building science and health science, along with recent advances in new building control, sensor technologies, and exposure assessment techniques, has now made enhanced multidisciplinary approaches to building research possible and necessary. This special issue on Healthy Buildings of the Journal of Exposure Science and Environmental Epidemiology highlights some novel studies related to better understanding the building related factors that influence health in buildings. Based on the major themes of the publications herein, we offer here our perspective on the (near) future possibilities of healthy buildings in the following six areas:

(1) Buildings as health promoting environments

(2) Ventilation standards designed for health

(3) Material health and chemical reduction

Guest Editors: Special Issue on Healthy Buildings

Joseph G. Allen

jgallen@hsph.harvard.edu

$\triangle$ Michael S. Waring

msw59@drexel.edu

1 Department of Environmental Health, Harvard T.H. Chan School of Public Health, Boston, MA, USA

2 Department of Civil, Architectural and Environmental Engineering, Drexel University, Philadelphia, PA, USA
(4) Change from static to dynamic evaluation

(5) Nature and indoor ecology

(6) Equity in research

We are entering an era in which buildings should go beyond a "do no harm" paradigm and instead promote high performance environments so as to focus on maximal benefit to occupants. Studies have begun to demonstrate the available positive benefits to occupant well-being and productivity. Research in this area focused on changes in outcomes such as increased work performance or reduced absenteeism in office environments with increasing ventilation rates, with studies conducted in real environments. Studies are also utilizing simulated indoor environments to demonstrate the drivers of observed cognitive improvements owing to changes in indoor air quality or comfort. Operation of buildings using multiobjective optimization frameworks could allow dynamic control that balances outcomes such as health, productivity, pollutant exposure, comfort, and energy use over various time horizons of interest.

Ventilation is the replacement of stale indoor air with outdoor air, and it is usually due to uncontrolled leakage in residences and forced air systems in offices, commercial, and public buildings. Typically, ventilation in nonresidential buildings is set to target minimum requirements for acceptable indoor air quality. As such, outdoor air is supplied in a coarse manner at a fixed rate designated by a design occupancy number, potentially also with an economizing cycle. However, ventilation can and should be provided in smarter ways, and most buildings could be easily retrofitted to provide dynamic zonal ventilation control based on occupancy estimations in conjunction with desired outcomes. Though it would require more infrastructure, personalized ventilation allows each occupant to control their own microenvironment, generating higher comfort acceptability. The costs of additional equipment should be weighed against the benefits to worker health and performance. During certain times of the year and in certain 
climates, natural ventilation may be used, which will provide energy reductions from using less fan energy, and less energy to heat or cool the outside air. Some studies have shown a wider comfort band for occupants in naturally ventilated buildings. However, more investigation is required into whether natural ventilation, which has much less opportunity for active particle or gaseous filtration, is worth any associated energy reductions. Finally, seasonality should be considered in building operation, so that mechanical systems can be operated to protect occupants against infectious diseases, such as using higher ventilation rates or relative humidity levels during months with high flu incidences.

In addition to controlling exposures through the mechanical system, renewed emphasis on the materials we put into our buildings is needed. Much research to date in the healthy building space has focused on exposures with acute effects that are quickly detectable and perceptible (thermal health, VOCs, noise, lighting). However, many classes of chemicals found in building materials and consumer products are SVOCs that cause a range of subchronic and chronic effects, many of which may be subclinical. These include endocrine-disrupting flame retardant chemicals, halogenated antimicrobials, and perand polyfluorinated alkyl substances (PFAS; colloquially called 'Forever Chemicals'), with other 'new' chemicals being discovered each year, and the woefully understudied field of nanomaterials. From a practitioner standpoint, there is progress on this front in terms of awareness, as 'material health' is becoming a new focus in building standards. Chemical 'red lists' and product transparency and disclosures are gaining momentum, and are a good start, but the future of healthy buildings requires a move toward the precautionary principle, where chemicals in products are thoroughly evaluated pre market.

New low-cost environmental sensors as well as sophisticated time of flight analyses are giving unprecedented insight into the dynamic nature of indoor environments. Exposure science is rapidly moving from studying static conditions (e.g., single pollutant, 8-h time-weighted average measures) to approaches where we can measure and model the true dynamic conditions in the space (e.g., real-time measures of multi-pollutant reactions with high spatial and/or temporal resolution). The massive reams of data produced by ubiquitous sensors in buildings and our ability to measure complex interactions - on windows, on floors, and on people-are giving us unprecedented insight into what's happening in our buildings, while also begging the question-"What does it all mean?" The role of the exposure scientist and environmental epidemiologist has never been needed more. The future ubiquity of 'smart' buildings powered by IoT and low-cost, real-time sensors will require input from our field to get to the fundamental question we've been studying for decades-"What is the magnitude, duration, and frequency of exposures, and how does this impact health?" In other words, this field needs to answer the question the public is asking_-"Why should we care?"

The interaction of physical and chemical processes in buildings has been more deeply studied than the role of biological factors. Early research in the biologicals domain focused on the role of the building as it relates to infectious disease transmission (e.g., influenza, tuberculosis). The need for this research remains in light of the potential for new and old pandemics, and for continued exploration of how building systems may play a role in mitigating transmission. New domains related to indoor biology and microbial ecology are still relatively nascent, but are exciting and fast-growing fields of research. At the microscale, advancements in metagenomics tools has just begun to give us insight into the microbes that live in us, on us, and surround us in our buildings. At the macroscale, 'indoor nature' studies are becoming more prevalent as new technologies like virtual reality and eye-tracking sensors allow us to study the impact of 'biophilic design' on stress, cognitive function, and well-being.

We believe that the most important aspect of this burgeoning healthy buildings movement is ensuring that the research and its application benefits everyone, everywhere. A future of healthy buildings that is confined to a select few would be a gross failing. Researchers must play an important role here by creating studies that include: globally diverse buildings and exposures; races and ethnicities that have been historically under-represented in research; a focus on the unique needs, physiology, and preferences of women; and exploring the entirety of the reproduction and life cycles, from pre-conception through advanced aging.

\section{Compliance with ethical standards}

Conflict of interest The authors declare that they have no conflict of interest.

Publisher's note Springer Nature remains neutral with regard to jurisdictional claims in published maps and institutional affiliations. 